\title{
Simultaneous Estimation of Chords and Musical Context from Audio
}

\author{
Matthias Mauch*, Graduate Student Member, IEEE, and Simon Dixon \\ Queen Mary University of London, Centre for Digital Music, \\ School of Electronic Engineering and Computer Science, \\ Mile End Road, E1 4NS. London, UK \\ \{matthias.mauch, simon.dixon\}@elec.qmul.ac.uk \\ phone: +44 207882 5528, fax: +44 2078827997
}

\begin{abstract}
Chord labels provide a concise description of musical harmony. In pop and jazz music, a sequence of chord labels is often the only written record of a song, and forms the basis of so-called lead sheets. We devise a fully automatic method to simultaneously estimate from an audio waveform the chord sequence including bass notes, the metric positions of chords, and the key. The core of the method is a 6-layered dynamic Bayesian network, in which the four hidden source layers jointly model metric position, key, chord, and bass pitch class, while the two observed layers model low-level audio features corresponding to bass and treble tonal content. Using 109 different chords our method provides substantially more harmonic detail than previous approaches while maintaining a high level of accuracy. We show that with $71 \%$ correctly classified chords our method significantly exceeds the state of the art when tested against manually annotated ground truth transcriptions on the 176 audio tracks from the MIREX 2008 Chord Detection Task. We introduce a measure of segmentation quality and show that bass and meter modelling are especially beneficial for obtaining the correct level of granularity.
\end{abstract}

Index Terms-chord transcription, music signal processing, dynamic Bayesian networks (DBN)

EDICs Category: AUD-ANSY

\section{INTRODUCTION}

A chord is defined as the simultaneous sounding of two or more different notes. Accompaniment of jazz and popular music is based on progressions of chords and is rarely written out as complete sheet music. Instead, musicians usually rely on lead sheets [1]. A lead sheet typically contains the melody written on traditional staves with time and key signature, along with chord symbols over the staves and the nominal bass note for the chord (if different from the root note), as illustrated in Figure 1. The chords found in lead sheets are an abstraction of what is actually played in a performance of the song, since often a precise replication of the original is unnecessary, or even unwanted. In recent years, the popularity of lead sheets has been underpinned by the success of the commercial software Band in a Box ${ }^{1}$ and its non-commercial contender $M M A^{2}$, both designed to generate musical accompaniment from a representation very similar to a traditional lead sheet.

The underlying motivation of our research is to use automatic chord recognition to produce lead sheets. In the remainder of this section we motivate our design choices derived from this aim, and provide a summary of previous approaches. Figure 2 shows an overview of our system, and the details of the method are given in the two following sections: Section II explains how we extract bass and treble chroma features from audio, while Section III details the topology and parameter settings of the novel dynamic Bayesian network. Section IV provides comparative evaluations of our methods, followed in Section V by conclusions and a discussion.

\section{A. Objectives of this Work}

Our aim is that eventually musicians will be able to use automatically generated lead sheets in the same way as they have been using the traditional, hand-annotated variant. The first requirement derived from this motivation is to provide transcriptions of the musical parameters chord, key, bass, and metric position. Secondly, similar to human music listening, the interdependence of these musical parameters should be modelled, and inference on them should be simultaneous. For example, chords are interpreted according to the key, while at the same time the key can be understood as a product of the chords. Raphael calls this the "chicken and egg problem" [3, p. 659], and strongly argues for the simultaneous estimation of parameters for cases in which such interdependence

\footnotetext{
${ }^{1}$ http: //www.pgmusic.com/

${ }^{2}$ http://www.mellowood.ca/mma/
} 


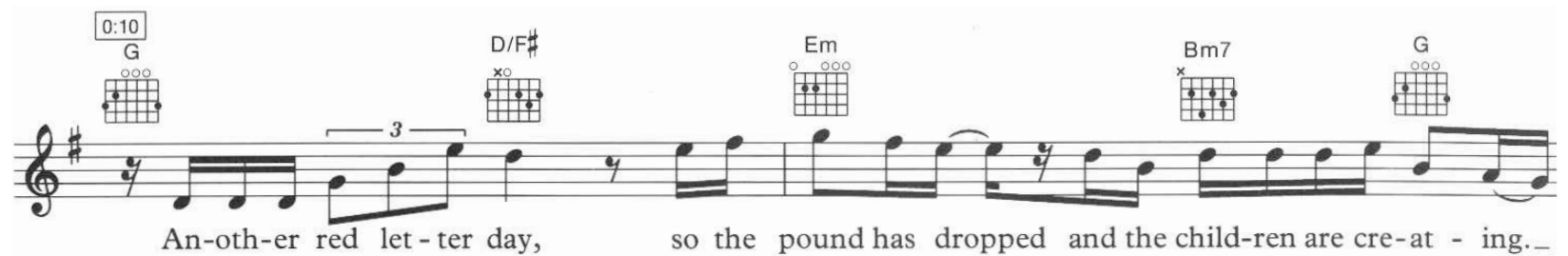

Fig. 1. Pop music lead-sheet: Excerpt of Friends Will Be Friends (Deacon/Mercury) taken from [2]. Chords are represented both by chord labels and the corresponding guitar fingering. The number in a box denotes the physical time. The bass is represented only implicitly in the chord labels.

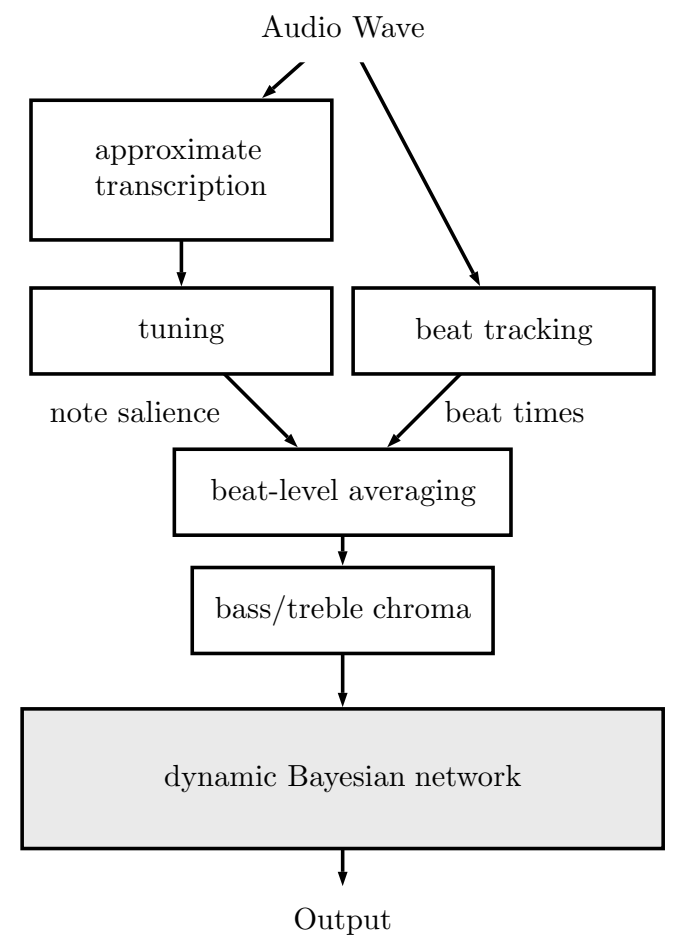

Fig. 2. A schematic overview of our method (see Sections II and III). White boxes represent the chroma extraction sub-methods.

arises. Finally, to do justice to the actual complexity of music, more specific chord labels are needed than have been used in previous automatic chord transcriptions. The choice of level of detail is difficult. On the one hand, the MIREX Chord Detection task [4] features only the two chord types major and minor. On the other hand, the chords actually used in pop songs are often much more complex (the software MMA has more than 100 chord types, i.e. 1200 chords). Our choice of 109 chords as detailed in Section III is by no means definitive, but much broader than has previously been attempted.

\section{B. Previous Work}

The foundation for a large majority of current methods for chord extraction is a low-level feature called the chroma vector (also, pitch class profile). The chroma vector is a twelve-dimensional vector of real numbers representing the energy or salience of the twelve pitch classes $(\mathrm{C}, \ldots, \mathrm{B})$, which amounts to considering pitch while suppressing the height dimension [5, p. 159]. Much like a spectrogram describes the spectral content of a signal over time, the chromagram is a sequence of chroma vectors that describes the pitch class content of an audio signal over time. Since its first use for chord extraction [6] the chromagram has also been used for other applications including key finding [7] and audioto-score synchronisation [8]. There are many ways to calculate chromagrams, for an introductory overview see [9]. For chord estimation, the quality of the chromagram has been improved by (automatic) tuning to the reference frequency [10] and median smoothing [11], removal of harmonics [12], and noise attenuation [13]. We combine some of these approaches in our own chromagram extraction algorithm ( $(\mathrm{II}-\mathrm{A})$.

To robustly infer chords from a chromagram, several temporal smoothing algorithms have been proposed to suppress short-term deviations from the chord. Examples are median filtering [10], hypothesis search [14], and hidden Markov models (HMMs) [15].

Statistical time series modelling in the music computing community has often been restricted to HMMs. Other approaches include graphical modelling for chord transcription from symbolic data [16], and conditional random fields [17]. There are numerous examples in which HMMs have been used to model and estimate the context of chords. Lee and Slaney [18] perform several HMM inference runs with different, key-dependent chord transition probabilities to implicitly determine the key of the piece in addition to the chords. A different HMM for key estimation from existing chord progressions has been proposed by Noland and Sandler [19]. Previously we integrated bass features into an HMM [20]. In pieces 
of music for which a beat-segmentation is known, an HMM can be used to perform a simultaneous estimation of the metric position of the beats and chords [21].

For more semantic flexibility than HMMs natively model (i.e. one hidden random variable and one observed random variable per time step), Leistikov [22] proposed the use of dynamic Bayesian networks as a way of modelling notes and their context in symbolic data. This allows for a more intuitive modelling process and an increase in inference efficiency. In the audio domain however DBNs have been used only in melody-tracking [23]; we are not aware of any previous applications of DBNs for the estimation of higher-level features such as chords.

The mentioned chord detection papers have in common the use of a very limited number of different chord types. For example, Lee and Slaney [18] choose to model three chord types (major, minor, diminished), leading to 36 different chords.

The novelty of the present work is that it integrates in a single graphical model pieces of musical context that had previously been assessed only separately. Keys, chords, metric position and bass pitch class can now be estimated simultaneously using the efficient inference techniques available for DBNs. We also increase the amount of output detail with respect to existing models, in particular, we increase the number of output chord types.

\section{Chromagram Calculation}

The aim of low-level processing in our case is to transform the audio input data into a representation which the high-level "musical" model can process. This representation consists of two different beat-synchronous chromagrams, one for the bass frequencies, and one for the treble frequencies, motivated by the importance of the bass note in harmony (see also Section III-D). In this section we explain how we obtain a note salience representation (or approximate transcription), how it is tuned and wrapped to chromagrams, and how it is finally averaged over beats.

\section{A. Note Salience}

Since the desired robust note transcription from complex audio remains an unsolved problem, we attempt an "approximate" transcription, which we refer to as note salience. The input files are monophonic wave files, lowpass filtered and downsampled to $f_{s}=11025 \mathrm{~Hz}$. We calculate the amplitude spectra $\chi$ of the wave using a Hamming window (length 2048 samples, i.e. $\approx 0.19$ seconds) with a hop size of $\Delta_{h}=0.05$ seconds.
The salience representation is based on a dictionary of complex tones covering the notes D1 (MIDI note $25, f_{0} \approx 37 \mathrm{~Hz}$ ) to $\mathrm{C} 6$ (MIDI note $84, f_{0} \approx 1047$ $\mathrm{Hz}$ ) in $1 / 3$ semitone steps. We synthesise the $m$ th tone with frequency $f_{0}^{m}$ as the weighted sum of its first four harmonics

$$
\begin{array}{r}
y_{m}(t)=\sum_{k=1}^{4} r^{k-1} \sin \left(2 \pi \cdot \frac{t}{f_{s}} \cdot k f_{0}^{m}\right), \\
t=1, \ldots, 2048 .
\end{array}
$$

We adopted a harmonic roll-off parameter $r=0.6$ in (1) from Gomez [7]. The amplitude spectra $M_{m}^{c}$. of these complex tones are obtained from $y_{m}(t)$ in the same way as those of the input files and appear as rows in the pattern matrix $M^{c}$. If $\chi_{j}$ denotes the amplitude spectrum of frame $j$, the product

$$
S_{j}^{c}=M^{c} \cdot \chi_{j},
$$

can be interpreted as the salience of the complex tones at frame $j$. In order to attenuate the salience at subharmonics introduced by using the complex tone pattern approach, we require that the energy at the fundamental frequency of the $m$ th tone be high. To that end we calculate a second dictionary matrix $M^{s}$ of simple tones using only the first term in the sum (1). The corresponding salience matrix $S^{s}$ is obtained analogously to $S^{c}$ in Equation (2) and subsequently convolved with a Laplacian kernel $(-1,-1,4,-1,-1)$ to amplify spectral peaks. Negative values are set to zero. The element-wise product

$$
S=S^{c} \otimes S^{s}
$$

combines the two matrices and yields a salience description for every note at every time frame.

\section{B. Tuning and Chroma Mapping}

Having three note salience values per semitone enables us to detect the tuning of a song. This is relevant because songs are not always recorded in standard 440 $\mathrm{Hz}$ tuning. We assume that the tuning frequency remains the same throughout each song. We use a tuning technique similar to the one used by Dressler and Streich [24]. The tuning is interpreted as an angle $\tau \in(-\pi, \pi]$, which corresponds to a tuning of

$$
2^{\tau /(12 \cdot 2 \pi)} \cdot 440 \mathrm{~Hz} .
$$

Hence, the three salience values pertaining to each semitone represent tunings

$$
\tau_{k}=\frac{2 \pi k}{3}, \quad k \in\{-1,0,1\}
$$




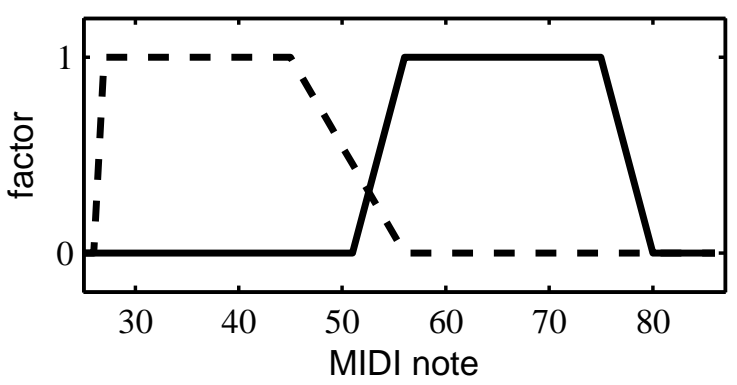

Fig. 3. Treble (solid line) and bass (dashed) templates $g$. These are used in (7) when calculating chromagrams from the note salience values.

We add the respective salience values over time, and over the note range,

$$
E_{k}=\sum_{j=1}^{T} \sum_{(m-k) \bmod 3=0} S_{m j}, \quad k \in\{-1,0,1\},
$$

and retrieve an estimate of the tuning by calculating the angle

$$
\hat{\tau}=\angle\left(\sum_{k=-1}^{1} E_{k} \cdot \exp \left\{\tau_{k} \sqrt{-1}\right\}\right) .
$$

We update $S$ by linear interpolation so that the centre bin of a semitone corresponds to $\hat{\tau}$ and then sum the three tone saliences pertaining to the same semitone to obtain the semitone-spaced salience matrix

$$
S_{k j}^{\mathrm{s}}=\sum_{\lceil m / 3\rceil=k} S_{m j} .
$$

The matrix $S^{\mathrm{s}}$ is subsequently median-filtered [11] in the time direction with a filter length of 9 frames $(0.45$ seconds). To obtain the treble chromagram $x^{*}$, the note salience $S^{\mathrm{s}}$ is "wrapped", i.e. note saliences that belong to the same pitch class are summed,

$$
x_{i j}^{*}=\sum_{(k-i) \bmod 12=0} S_{k j}^{\mathrm{s}} \cdot g_{k}, \quad i=1, \ldots, 12,
$$

weighted by the template $g$ illustrated in Figure 3, which discards bass and very high treble notes. The bass chromagram is analogously obtained using different weights $g$ to discard notes in the treble range.

\section{Averaging over Beats and Normalisation}

Beat, or "tactus", represents the main regular pulse in a piece of music [25, p. 71]. In order to segment the audio into musically meaningful chunks we use an automatic beat-tracking algorithm [26]. The system extracts beat
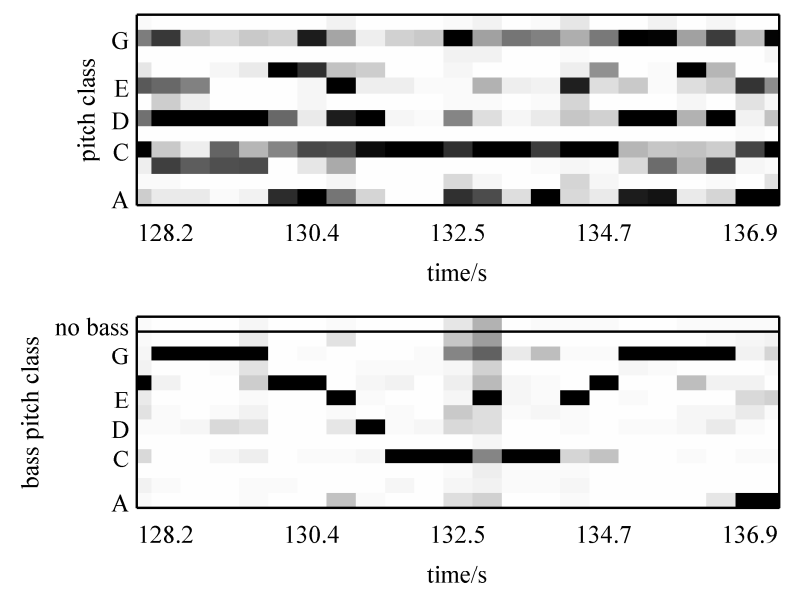

Fig. 4. Example treble and bass chromagrams generated from the song Let It Be (Lennon/McCartney).

times $0<t_{0}<\ldots<t_{N}$. We take the median (over time) of the chromagram frames within each beat,

$$
x_{i j}=\operatorname{median}_{t_{j} \leq\left(j^{\prime} \cdot \Delta_{h}\right)<t_{j+1}} x_{i j^{\prime}}^{*} .
$$

A measure of chroma flatness is computed to express the salience of "no bass note" and becomes a 13th dimension to the bass chromagram,

$$
x_{13, j}=\left(12 \cdot \max _{i} S_{i j} / \sum_{i^{\prime}=1}^{12} S_{i^{\prime} j}\right)^{-2} \in\left[\frac{1}{144}, 1\right] .
$$

Both beat-quantised chromagrams-including the additional bass bin-are subsequently normalised according to the maximum norm [7, p. 79], i.e. every bin value is given relative to the most salient bin of the same frame, see Figure 4.

\section{Network MOdeL}

A Bayesian network (BN) is a joint distribution of several random variables. It is called a "network" because its dependency structure can be represented using a directed acyclic graph. Every node represents one random variable ${ }^{3}$. A directed edge represents a direct dependency; it points at the node that directly depends on the node from which the edge originates. This duality of the graph and the joint distribution allows very intuitive modelling as detailed in this section. The requirement of the graph to be acyclic means that there is no dependency

\footnotetext{
${ }^{3}$ We will use the two expressions node and random variable interchangeably.
} 


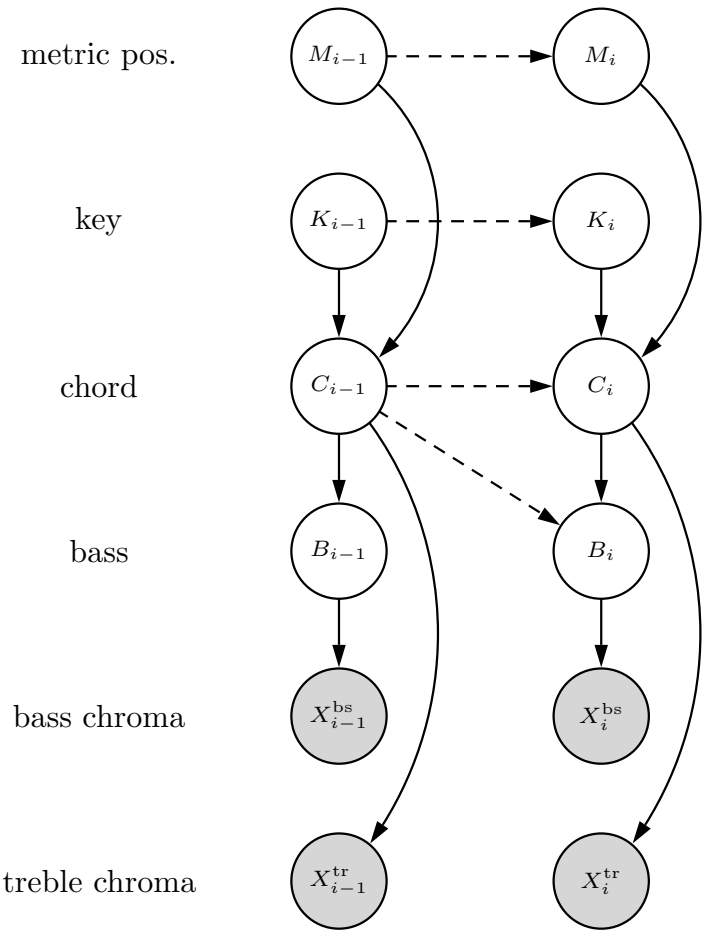

Fig. 5. Our network model topology, represented as a 2-TBN with two slices and six layers. The clear nodes represent random variables, while the observed ones are shaded grey. The directed edges represent the dependency structure. Intra-slice dependency edges are drawn solid, inter-slice dependency edges are dashed.

"short circuit", so a random variable is never its own descendent.

To model time series with BNs, dynamic Bayesian networks (DBNs) are used [27]. A DBN can be thought of as a succession of simple BNs. The succession is assumed to be Markovian, and time-invariant, i.e. the model can be described recursively by defining only two slices [28]: one "initial state" slice and one "recursive" slice. Such models are also called 2-slice temporal Bayesian networks (2-TBN). Note that any DBN could equivalently be modelled as an HMM, comprising the different state variables of the DBN in a single (very large) state variable. As a result, modelling of the adequate HMM is less intuitive and inference can be much slower [27].

In the DBN topology as shown in Figure 5 discrete nodes model the states of metric position, key, chord, and bass pitch class, and continuous nodes model bass and treble chroma. Our DBN is a generative model, i.e. some state configuration sequence of the hidden source nodes is assumed to have generated the observed data (chromagrams). This assumption allows us to use
Bayesian reasoning to infer the state sequence from the data [22, p. 96]. We use the Bayes Net Toolbox [29], which implements diverse inference and learning methods, to model the data and perform the inference.

To complete the definition of the network the conditional probability distributions (CPD) of the random variables need to be specified, providing a good approximation of how beats, keys, chords and bass interact. Since we do not have any preconception of the initial metric position, key, chord or bass pitch class of a piece, all initial nodes are set to a uniform distribution. The following subsections will detail the CPDs of the recursive nodes on the right hand side of the 2 -TBN depicted in Figure 5. Like Leistikow [22] we choose to map expert musical knowledge onto a probabilistic framework, rather than learning parameters from a specific data set. In a complex model such as the one presented in this section, the decisions regarding parameter binding during learning, and even the choice of the parameters to be learned pose challenging research questions, which we plan to address in future work, while focusing here on the definition and evaluation of the expert model.

\section{A. Metric Position}

Western music is usually grouped in bars, each containing a number of beats. In much popular music, there are four beats per bar throughout a piece, and our model assumes this case. The first beat (metric position 1) in a bar is followed by the second (metric position 2), and so on, until after the fourth the next bar starts on metric position 1 . Hence, the node $M_{i}$ has four states to represent the metric position of the current beat. We use pieces of music in which occasional beat tracking errors or compositional irregularities in the music are frequent, hence we have to allow for the small probability $\varepsilon=0.05$ of deviation from the normal succession of beats. Since node $M_{i}$ depends only on node $M_{i-1}$, the conditional distribution $P\left(M_{i} \mid M_{i-1}\right)$ can be represented as a transition matrix with two dimensions,

$$
\left(\begin{array}{cccc}
\varepsilon / 2 & 1-\varepsilon & \varepsilon / 2 & 0 \\
0 & \varepsilon / 2 & 1-\varepsilon & \varepsilon / 2 \\
\varepsilon / 2 & 0 & \varepsilon / 2 & 1-\varepsilon \\
1-\varepsilon & \varepsilon / 2 & 0 & \varepsilon / 2
\end{array}\right)
$$

Each row represents a state of $M_{i-1}$, every column a state of $M_{i}$. The same information can be written as a 


$$
\begin{array}{llllllllllllll}
\text { C } & \text { C\# } & \text { D } & \text { Eb } & \text { E } & \text { F } & \text { F\# } & \text { G } & \text { Ab } & \text { A } & \text { Bb } & \text { B }
\end{array}
$$

Fig. 6. Key: C major/A minor key. Pitch classes in shaded squares are the ones belonging to the key. To obtain the other keys, the pitch classes are "rolled" accordingly (circular shift).

conditional probability distribution,

$$
P\left(m_{i} \mid m_{i-1}\right)=\left\{\begin{array}{cl}
1-\varepsilon & \text { if }\left(m_{i}-m_{i-1}\right) \bmod 4=1, \\
\varepsilon / 2 & \text { if }\left(m_{i}-m_{i-1}\right) \bmod 4 \in\{0,2\} \\
0 & \text { otherwise }
\end{array}\right.
$$

\section{B. Key}

The node $K_{i}$ represents the key state. The knowledge of the key and key changes can have two benefits: improving the stability of the chord estimation by making off-key chords less probable, and providing a means of setting the key signature in a score. We choose to model 12 keys, each of which corresponds to a major/relative minor key pair, which is enough to cover all key signatures, since any major and the corresponding relative minor key share a key signature. Relative to the root pitch class, every key has a diatonic profile; an example is depicted in Figure 6. To model the key we only need to express that the key is expected to remain the same with a high probability of 0.98 , i.e. we assume that at any beat the key changes with a probability of 0.02 :

$$
P\left(k_{i} \mid k_{i-1}\right)=\left\{\begin{array}{cl}
0.98 & \text { if } k_{i-1}=k_{i}, \\
(1-0.98) / 11 & \text { otherwise. }
\end{array}\right.
$$

The behaviour of the key node only describes the rate of change of keys. The way in which the key acts upon the chord is coded into the chord CPD as detailed in the following subsection.

\section{Chord and Treble Chroma}

The chord nodes $C_{i-1}$ and $C_{i}$ together with the respective treble chroma nodes $X_{i}$ and $X_{i-1}$, take a central place in our model. We use a pool of $N_{\mathrm{C}}=109$ chords:

- $7 \times 12$ in root position: major (shorthand $\left.{ }^{4}: m a j\right)$, minor (min), major 7th (maj7), major with a minor 7th (7), major 6th (maj6), diminished (dim), augmented (aug),

- $2 \times 12$ major chords in first and second inversion (maj/3 and $\mathrm{maj} / 5)$, and

- 1 "no chord" (N).

${ }^{4}$ We use the shorthand notation as proposed in [30], but omit the colon as in $\mathrm{C}: \mathrm{maj} 7$.

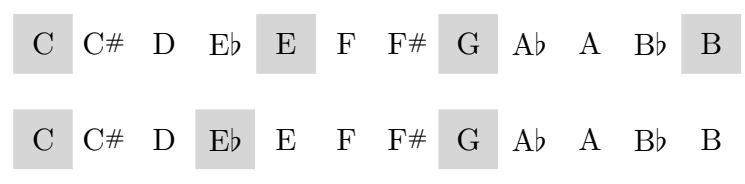

Fig. 7. Chord examples: $\mathrm{Cmaj} 7$ and $\mathrm{Cmin}$ chords. The shaded squares denote the pitch classes belonging to the chord. To obtain the same chord type with a different root, the chord is "rolled" (circular shift).

To keep calculations feasible and prevent overspecification we have refrained from including yet more chords, for example sus 4 and min 7 . However we believe that this choice, in particular the 7 chord (suggesting a functional difference to maj), and the inversions offer a great increase of information when compared to a smaller set of maj, min, dim, and aug chords.

First, let us consider the treble chroma node $X_{i}$ (Figure 5). Following Harte's chord definitions [30], the quality of a chord is expressed by the pitch classes it contains (see Figure 7). This should be reflected in the treble chroma the chord generates. As has been explained in Section II, the chroma features $x_{i} \in[0,1]$ are normalised by the maximum norm, so high values will be close to one, and-ideally-low values close to zero. The probability density $P\left(X_{i} \mid c_{i}\right)$ of the chroma node given a chord should monotonically increase with any of the chord pitch class saliences increasing. It should monotonically decrease with any of the non-chord pitch class saliences increasing. We model this behaviour as a 12-dimensional Gaussian random variable in which the mean vector has zeros at the elements representing nonchord pitch classes and ones at elements representing the chord pitch classes, see Figure 8 . We choose a diagonal covariance matrix in which all diagonal elements are set to $\sigma^{2}=0.2$. A rigorous estmimation of variance values is left to future work. Note that due to the chroma normalisation, a flat chroma vector will contain only ones. Therefore, we define $\mathrm{N}$ (no chord) as including all pitch classes.

We have described the treble chroma node, which depends only on the chord node. The chord node itself, $C_{i}$, depends on the previous chord node $C_{i-1}$ as well as the current metric position node $M_{i}$ and the current key node $K_{i}$. This configuration allows us to model that

- a chord change is likely at the beginning of a bar (metric position 1), less likely in the middle of a bar (position 3), and even less likely at the remaining metric positions 2 and 4 ,

- a chord is more likely the fewer non-key pitch classes it contains. 


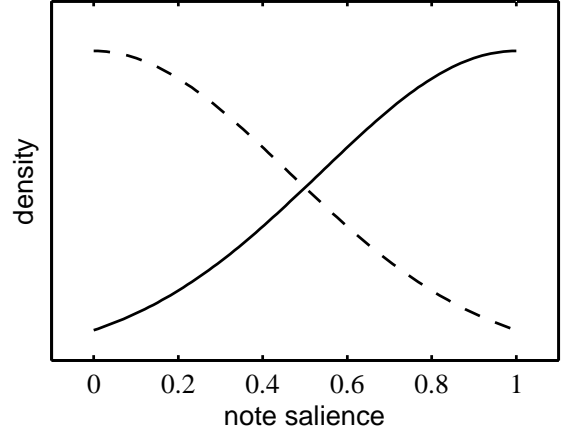

Fig. 8. Treble chroma node: distribution of single elements of the 12dimensional Gaussian, monotonically increasing curve for chord pitch classes, monotonically decreasing curve (dashed) for non-chord pitch classes.

Accordingly, we factorise the probability as

$$
P\left(c_{i} \mid c_{i-1}, m_{i}, k_{i}\right)=P\left(c_{i} \mid c_{i-1}, m_{i}\right) \cdot P\left(c_{i} \mid k_{i}\right),
$$

in which the first factor describes the dependency of a chord change on the metric position. Let the vector

$$
a=(0.5,0.1,0.4,0.1)
$$

contain the probabilities of a chord change at metric positions 1 to 4 , then

$$
P\left(c_{i} \mid c_{i-1}, m_{i}\right)=\left\{\begin{array}{cl}
a_{m_{i}} /\left(N_{\mathrm{C}}-1\right) & \text { if } c_{i-1} \neq c_{i}, \\
\left(1-a_{m_{i}}\right) & \text { otherwise }
\end{array}\right.
$$

where $N_{\mathrm{C}}$ is the number of chords. The second factor in (12) describes how likely a chord is, conditional on the key. Perceptual chord ratings in a key context are available for maj, min, and dim chords [31], but not for the more complex chords we consider. We introduce an expert function

$$
f\left(c_{i}, k_{i}\right)=\frac{1}{\#\{\text { non-key chord notes }\}+c}
$$

that can express a rating for any kind of chord. To determine the smoothing parameter $c$ we use as a reference the maj chord subset of the mentioned chord ratings (Figure 9), interpreted as probabilities [19]. To obtain a function $f$ that approximates the ratings best, we minimize with respect to $c$ the Jensen-Shannon divergence between the chord ratings and the corresponding ones obtained from the function $f$. The resulting value of $c=4.83$ is then used for all chords. For instance, the $\mathrm{Cmaj} 7$ chord depicted in Figure 7, in the key of $\mathrm{C}$ major has $f(\operatorname{Cmaj} 7, \mathrm{C}$ major $)=\frac{1}{0+c} \approx 0.21$, whereas for the $\mathrm{Cmin}$ chord in the same figure, $f(\mathrm{Cmin} \mid \mathrm{C}$ major $)=$ $\frac{1}{1+c} \approx 0.17$ because $\mathrm{E} b$ is not part of the $\mathrm{C}$ major key.

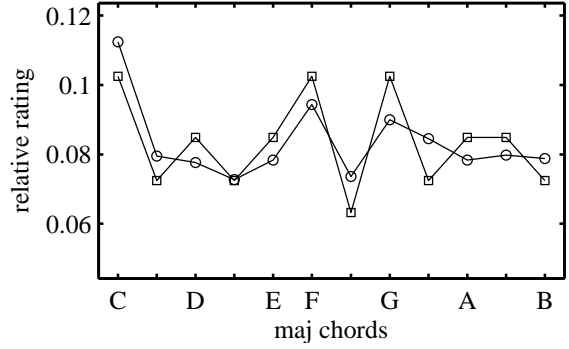

Fig. 9. Our $f$ chord-context ratings (denoted by $\square$ ) for major chords in a $\mathrm{C}$ major key context, compared to the Krumhansl profiles (o), both normalised by the $L_{1}$ norm.

The $f$ values are then normalised by a constant $\kappa$ such that

$$
P\left(c_{i} \mid k_{i}\right)=\kappa \cdot f\left(c_{i}, k_{i}\right)
$$

is a conditional probability distribution, i.e. for a fixed $k_{i}$ the probabilities sum to unity.

\section{Bass Pitch Class and Bass Chroma}

The bass pitch class plays a crucial role in the recognition of chords. Being at the bottom of the frequency range, it "anchors" the chord and makes the rest of the notes more easily interpretable. For instance, knowing whether the bass note is $\mathrm{C}$ or $\mathrm{E}$ can help disambiguate the chords $\mathrm{Cmaj} 7$ and Emin, which have very similar pitch class sets (namely, C, E, G, B and E, G, B).

A bass pitch class can be determined for every chord on a lead sheet. In chords written without further bass information, the bass pitch class is the same as the root note, otherwise the slash notation of the bass pitch class determines the bass pitch class. In Harte's syntax [30], an Fmaj chord has the bass note $F$, but the bass pitch class of its first inversion Fmaj/ 3 is A, where / 3 means that the bass note is the third above the root.

The bass chroma is modelled in much the same way as the treble chroma, by a Gaussian vector. Its number of dimensions is $13=12+1$, with 12 dimensions representing the bass pitch classes $C$ through $B$, and the thirteenth representing "no bass note". Since the bass is defined by just one note, every profile has only one element for which the mean value is set to 1 (rather than 3 or 4 in the case of chords), while the others are set to 0 . Usually only one bass note is played at any time, which implies that the pitch class played will more often have a normalised salience of 1 , and the other pitch classes will have saliences close to zero. Accordingly, we choose a lower variance value of $\sigma=0.1$.

Bass lines tend to include many different consecutive notes and pitch classes. The role of the chord bass 
pitch class becomes clear if one observes that in popular music the bass note is almost always present on the first beat of a chord. One popular bass player tutorial [32] confirms this: among the 207 example bass patterns covering styles Blues \& R'n'B, Soul, Motown/Atlantic Records, Funk, and Rock only 20 do not start with the bass pitch class. Allowing for some more variation than given in these examples, we estimate that the played and the chord bass note coincide on the first beat of the chord $80 \%$ of the time. To model this behaviour, we set the probabilities to

$$
P\left(b_{i} \mid c_{i-1} \neq c_{i}\right)= \begin{cases}0.8 & \text { if bass is chord bass } \\ 0.2 / 12 & \text { otherwise }\end{cases}
$$

As the chord continues, we still expect the "nominal" bass pitch class as the most likely option, but other pitch classes may be used as a bass note too, so we set the probabilities as follows:

$$
P\left(b_{i} \mid c_{i-1}=c_{i}\right)= \begin{cases}0.4 & \text { if bass is chord bass } \\ 0.6 / 12 & \text { otherwise }\end{cases}
$$

Note that while modelling essential properties of popular music in 4/4 time, the CPDs described in this section do not explicitly suppress or encourage particular key, chord or bass note transitions.

\section{EVALUATION}

Since chord labelling is not a well-defined classification task even for human musicians, the evaluation of automatic chord transcription is difficult. It has been common practice to use the relative correct overlap with respect to a ground truth annotation as an accuracy measure [4]. We would also like to stress that chord extraction from audio is a segmentation task as much as a classification task, and the similarity of ground truth and automatic segmentation should be taken into account. Both kinds of measures will be explained in this section, followed by the corresponding results.

\section{A. Performance Measures}

A segmentation of a song is a vector $B$ of one or more contiguous, non-overlapping intervals $B_{1}, \ldots, B_{N_{B}}$ such that $\bigcup B_{i}$ covers the whole song, and $T=\left|\bigcup B_{i}\right|$ is the length of the song, where vertical lines $|\cdot|$ denote the length of an interval. Let $B^{0}=\left(B_{1}^{0}, \ldots, B_{N_{0}}^{0}\right)$ be the given (ground truth) segmentation, and $B$ that obtained from an automatic algorithm. Similarly, let $L^{0}=\left(l_{1}^{0}, \ldots, l_{N_{0}}^{0}\right)$ be the ground truth class labels corresponding to $B^{0}$, and $L=\left(l_{1}, \ldots, l_{N}\right)$ those corresponding to $B$.
1) Relative Correct Overlap and MIREX Score: Rather than dealing with thousands of possible chords directly we break up the chords into classes, resulting in a partition $\mathcal{L}$. If the chord labels $l_{1}$ and $l_{2}$ are in the same class, they are called $\mathcal{L}$-equivalent, $l_{1} \sim l_{2}$. In the MIREX task, the chord labels are partitioned into $|\mathcal{L}|=25$ different classes: $12 \mathrm{~min}$ classes (each class comprises the chords whose labels contain min and which have identical roots, for example, Fmin $\sim$ Fmin 7), and 12 maj classes (each class comprises the chords whose labels do not contain min or $\mathrm{N}$ and which have identical roots), as well as the "no chord" class $\mathrm{N}$. We use the Iverson bracket as follows:

$$
\left[l_{1} \sim l_{2}\right]= \begin{cases}1 & \text { if } l_{1}, l_{2} \text { are } \mathcal{L} \text {-equivalent } \\ 0 & \text { otherwise }\end{cases}
$$

The relative correct overlap for one song is then defined as

$$
\mathcal{O}_{\mathcal{L}}=\frac{1}{T} \sum_{i=1}^{N_{0}} \sum_{j=1}^{N}\left|B_{i}^{0} \cap B_{j}\right| \times\left[l_{i}^{0} \sim l_{j}\right] .
$$

The MIREX score is the mean of $\mathcal{O}_{\mathcal{L}}$ over all songs. The choice of MIREX chord classes is very coarse, and for the further results we use a different $\mathcal{L}$ to differentiate the $|\mathcal{L}|=109$ chord classes that map most closely to the chords in the DBN.

The measure described above is necessarily biased towards the chord type that occupies most of the duration in a song or collection. In the case of the Beatles' music this is the maj chord type. To assess the method's performance on a specific chord subset $\mathcal{S} \subset \mathcal{L}$ the formula (20) changes to

$$
\sum_{l_{i}^{0} \in \mathcal{S}} \sum_{j=1}^{N}\left|B_{i}^{0} \cap B_{j}\right| \times\left[l_{i}^{0} \sim l_{j}\right] / \sum_{l_{i}^{0} \in \mathcal{S}}\left|B_{i}^{0}\right| .
$$

2) Segmentation Quality: The segmentation quality of a transcription with respect to the ground truth can be evaluated without taking chord labels into account. This is desirable because such a measure is less likely to suffer from the necessarily subjective chord interpretation of the ground truth annotator.

The measure we propose is based on the directional Hamming divergence ${ }^{5}$, which has been used in the context of image segmentation [33] and musical song segmentation [34]. For each interval in a segmentation, the directional Hamming divergence measures how much of it is not overlapped by the maximally overlapping segment of the other segmentation. Then the values over all intervals are summed. In mathematical terms,

\footnotetext{
5 also called directional Hamming distance
} 
given two segmentations $B^{0}, B$ we define the directional Hamming divergence as

$$
h\left(B \| B^{0}\right)=\sum_{i=1}^{N_{B}}\left(\left|B_{i}^{0}\right|-\max _{j}\left|B_{i}^{0} \cap B_{j}\right|\right) .
$$

It describes how fragmented $B$ is with respect to $B^{0}$. If we swap the two segmentations in equation (22), we obtain what has been called the inverse directional Hamming distance, a measure of how fragmented $B^{0}$ is with respect to $B$. The arithmetic mean of both, normalised by the length of the song is a symmetric measure for the dissimilarity of the two segmentations.

$$
H\left(B, B^{0}\right)=\frac{h\left(B \| B^{0}\right)+h\left(B^{0} \| B\right)}{2 T}
$$

It is desirable that an automatic transcription $B$ have low $H\left(B, B^{0}\right)$ against a ground truth segmentation $B^{0}$.

\section{B. Results}

We use Beatles chord transcriptions [30] as ground truth, and extract chromagrams from the corresponding original Beatles recordings. Several experiments are conducted to investigate the influence of choice of chord set, metric position, bass note, and key in our model. We choose among three different chord sets, namely

full the full chord set, consisting of all 109 chords introduced in Section III-C,

maj-min only the two chord classes maj and min, and the $\mathrm{N}$ class ( 25 chords), and

inv the set which extends the maj-min set by adding the first and second inversion major chords maj/3 and maj/5 (49 chords).

We also consider four different DBN configurations by enabling only specific nodes.

plain In the plain model, the metric position, key, and bass pitch class modelling is disabled, chord duration is modelled as a negative binomial distribution ${ }^{6}[20]$ with shape parameter 2 , and scale parameter $1 / 3$, corresponding to an expected chord duration of 4 beats.

$M \quad$ In the metric model $(M)$, metric position is fully modelled as described in III; bass and key are disabled.

$M B \quad$ In the metric-bass model $(M B)$, the bass pitch class node is additionaly enabled.

MBK The metric-bass-key model $(M B K)$ is the entire model as described in III.

We infer the most likely state sequence for the enabled discrete nodes using the Viterbi algorithm. Inference in

\footnotetext{
${ }^{6}$ the discrete analogue of a gamma distribution
}

\begin{tabular}{lrrrr}
\hline & \multicolumn{4}{c}{ MIREX score } \\
chord set & plain & $M$ & $M B$ & $M B K$ \\
\hline maj-min & 0.663 & 0.674 & 0.703 & 0.709 \\
inv & n/a & n/a & 0.716 & 0.712 \\
full & 0.654 & 0.662 & 0.704 & 0.709 \\
\hline BP MIREX & \multicolumn{4}{c}{0.661} \\
\hline
\end{tabular}

TABLE I

MIREX $\mathcal{O}$ SCORE RESULTS: MEAN RELATIVE CORRECT OVERLAP, AVERAGED OVER THE 176 SONGS USED IN THE 2008 MIREX TASK. FOR SIGNIFICANCE TESTS SEE SECTION IV-B1. BP MIREX

[15] IS THE BEST PERFORMING ALGORITHM IN THE ORIGINAL TASK.

the most complex model, the $M B K$ model with full chord set, is very memory-intensive, since the chord node would have to deal with $109 \times 4 \times 12 \times 109=570288$ states. We perform a preprocessing step to discard the 59 chords that appear least often among the locally bestfitting 10 chords at every beat, leaving us with 50 chords, which are still fully connected. Processing time does not exceed the song play time, for example, inference on the Beatles song You Won't See Me (Lennon/McCartney) with a play time of 202 seconds takes 104 seconds using the full-MBK model.

1) MIREX-Style Results: The MIREX score as defined in (20) is a good benchmark for comparing our algorithm to others', since the song-wise original MIREX task results are freely available ${ }^{7}$. To comply with the MIREX format, we have to map all our chords to the $24 \mathrm{maj}$ and min labels, plus one $\mathrm{N}$ label. All chords with a major third degree are mapped to the respective maj chord, all chords with a minor third degree to the respective min chord. Several versions of our algorithm (Table I) have a mean relative correct overlap of over 0.70 , i.e. they perform better than the best performances in the 2008 MIREX pretrained Audio Chord Detection task (Bello and Pickens [15] scored 0.66). To assess if the MIREX score difference between our best-performing model, inv-MBK, and Bello and Pickens's model are significant, we perform a one-way ANOVA analysis. The resulting $p$-value of 0.006 is very low, and hence we can be confident that our inv-MBK model performs significantly better. A further TukeyKramer multiple comparison test between all our models and Bello and Pickens's at 95\% confidence level based on the Friedman analysis of variance (see, e.g. [35]) confirms that the $M B K$ models all perform significantly better than Bello and Pickens's. To assess which of the variants of our model have a significant influence on the

\footnotetext{
${ }^{7} \mathrm{http} / / /$ www.music-ir.org/mirex/2008/results/chord/task1_results/ ACD.task1.results.overlapScores.csv
} 


\begin{tabular}{cccc}
\hline plain & $M$ & $M B$ & $M B K$ \\
0.186 & 0.176 & 0.167 & 0.166 \\
\hline
\end{tabular}

TABLE II

MEAN SEGMENTATION DIVERGENCE (EQUATION 23) OF DIFFERENT MODELS, USING full CHORDS. LOWER VALUES ARE BETTER. IMPROVEMENTS FROM plain TO $M$ AND FROM $M$ TO $M B$ ARE SIGNIFICANT (SEE IV-B2).

MIREX score, we consider only the full chords versions and perform a Tukey-Kramer multiple comparison based on the Friedman analysis of variance. We find that with 95\% confidence, each additionally added node achieves a significant improvement. We conclude that meter, bass, and key modelling all significantly contribute to better chord labelling in our model.

2) Segmentation Quality: We evaluate the segmentation quality according to the $H$ measure given in Equation (23) on all full chord versions (see Table II). The more complex models yield lower, i.e. better, segmentation scores. In fact, according to the TukeyKramer multiple comparison with a confidence level of $95 \%$, segmentation significantly improves by adding meter modelling to the plain model. Additionally adding bass modelling to the $M$ model brings about another significant improvement. Meter and bass modelling provide means of finding chord change positions at a level of granularity more closely related to manual annotations.

3) Chord Confusion: For the rest of our evaluation we will consider the full-MBK model as described in Section III and confine the analysis to 155 Beatles songs that do not explicitly violate the time signature assumption we made in our model. To investigate the method's performance on less common chords we use 109 specific chord classes (instead of the coarser MIREX classes), 12 for each of the chord types used in the model (see $\S \mathrm{III}-\mathrm{C})$, as well as N.

Figure 10 shows that the mean relative overlap score remains at a high overall level of 0.62 even with the much finer class partitioning. The ma $j$ and min chords are recognised most reliably, followed by "no chord" and aug and dim chords. The worse performance of $\operatorname{maj} 7,7$, and $\operatorname{maj} 6$ chords is not surprising, since these are maj chords with added notes. For instance, taking a closer look at the 7 chord reveals that the 7 chord is most frequently classified as the ordinary maj chord on the same root (Figure 10 (b)). Since reversely very few maj chords are incorrectly recognised as 7 chords (relative overlap is 0.026 ), the successfully recognised 7 chords add a new level of detail to chord recognition. The other three among the top five confusions in Figure 10 (b) are easily explained too, since they all share two

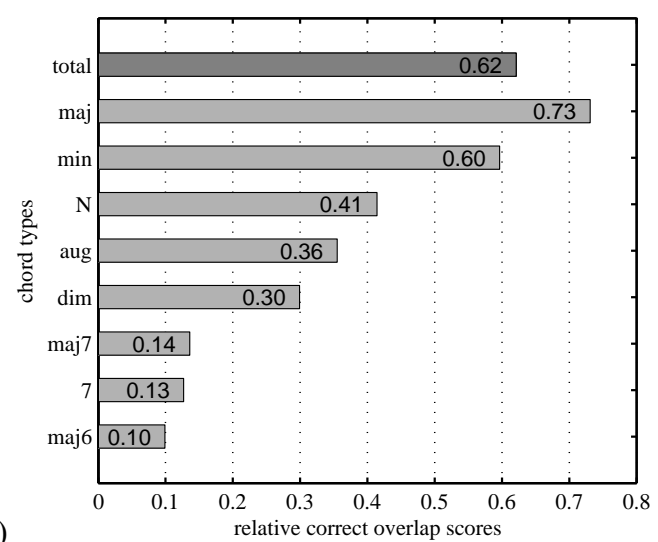

a)

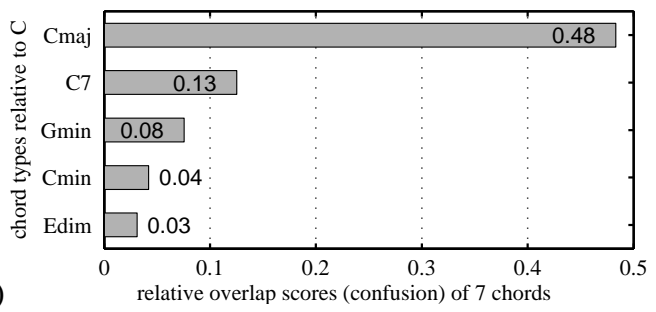

Fig. 10. Full chords $M B K$ : relative correct overlap scores for different chord types. Chords are considered correct if root note and chord class match, inversions of the same chord are considered equivalent. (a) shows chord type overlap, (b) details the most common matches for chord class 7 , with the correct chord itself ranking second.

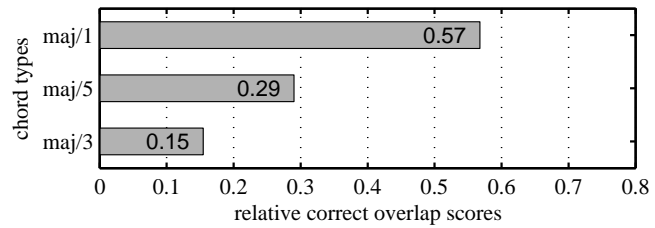

Fig. 11. Full chords $M B K$ : maj chord relative correct overlap (root note, chord class, and bass note match) for the different inversions.

or three pitch classes with the 7 chord.

The inversions of ma $j$ chords are of particular interest, since they show the impact of the bass note. Figure 11 shows that maj chords in root position score highest. Chords in first inversion are recognised as such only $15 \%$ of the time, but can still provide information that was not available with previous approaches, e.g. see Figure 12. Second inversion chords have an overlap score of $29 \%$.

4) Key Signature: We model only the key signature, i.e. 12 different major/minor pairs. For a given piece, we retrieve the main key signature (see $\S$ III-B) that is active most frequently. Our method correctly recognises $63 \%$ of the main keys signatures, which is not very high compared to state of the art key extraction algorithms [36], but acceptable since we do not explicitly model minor keys. Since the additional key information does 


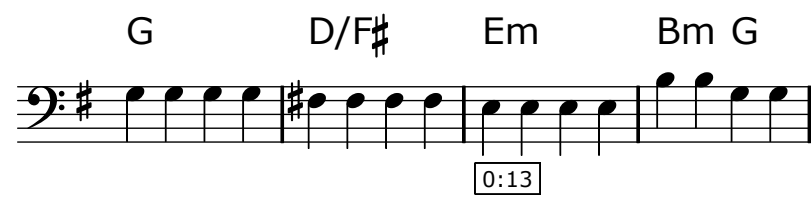

Fig. 12. Excerpt of an automatic output of our algorithm using the inv-MBK model for Friends Will be Friends (compare to Figure 1), music engraving by LilyPond. Physical time is displayed in the box. In the second bar, the Dmaj chord is correctly identified as being in first inversion. The key signature of $\mathrm{G}$ major is also correct. The notes in the staves represent the bass pitch class.

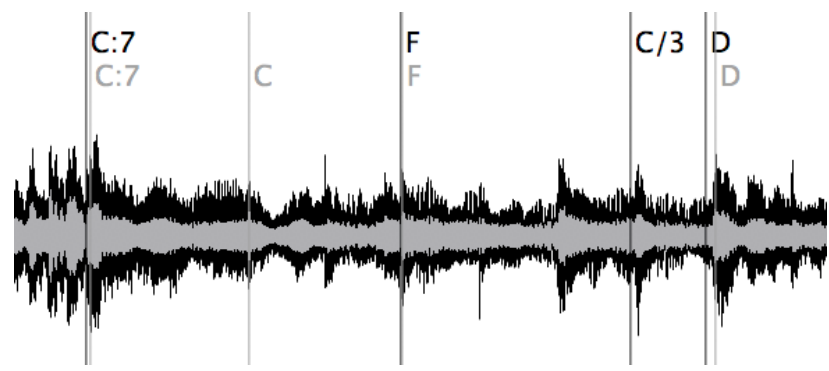

Fig. 13. Excerpt of Something (Lennon/McCartney), displayed in the free software Sonic Visualiser. The first (black) line of chords is the ground truth transcription, the lines below (grey chord symbols) are our automatic transcription, using full chords, metric position, bass, and key.

provide an increased performance in the MIREX score (see $\S I V-B 1$ ), we expect that future work on key modelling will result in further improvements.

5) Examples: Our system automatically generates LilyPond $^{8}$ source files and Sonic Visualiser ${ }^{9}$ XML files. The lead sheet depicted in Figure 12 is compiled from a LilyPond source file. Key, chord inversion, and the metric information provide a detailed notation that matches the official version from [2] depicted in Figure 1.

In Figure 13 an excerpt of the song Something (Lennon/McCartney), is displayed as loaded from an automatically created XML file into Sonic Visualiser (grey). For comparison we have additionally loaded the ground truth annotations (black). Note that while the ground truth correctly annotates the first two full bars of the example as $\mathrm{C} 7$, our method switches back to $\mathrm{Cma} j$ in the second bar. This happens because in the second bar the flat seventh that turns a $\mathrm{Cmaj}$ chord into a $\mathrm{C} 7$ is not present, but still assumed to continue by the annotator. This gives a qualitative explanation for the confusion of the 7 chord discussed in $\S \mathrm{IV}-\mathrm{B} 3$.

\footnotetext{
${ }^{8}$ http://lilypond.org/web/

${ }^{9} \mathrm{http}: / /$ www.sonicvisualiser.org/
}

\section{Conclusions and Future Directions}

We have presented a musically-informed dynamic Bayesian network for the automatic extraction of chord transcriptions from musical audio. The main novelty of this approach is simultaneous inference of metric position, key, chord, and bass pitch class, which reflects the natural inter-dependence of these entities. With 109 chord classes, the model provides a higher level of detail than previous approaches.

The method presented achieves a mean correct overlap score of $71 \%$, and significantly outperforms all systems tested in the 2008 MIREX task for pretrained chord detection. We compared 10 different variants of our algorithm and show that each additional musical parameter significantly improves the method's performance. The greatest enhancement is achieved by additional bass modelling. While aiding the correct identification of chords, the key estimation itself has performed slightly worse than anticipated. The high number of chords provides new musical information, without decreasing the performance of the method.

As a complement to the correct overlap evaluation method, we have introduced a measure of chord segmentation quality which provides a measure of how well the locations and granularity of chord changes resemble those of the ground truth. Our results show a significant improvement in segmentation quality due to modelling of metric position and bass.

Taking the present expert system as a point of departure, we believe that careful probabilistic learning could yield even better results, despite inevitably being specific to the music collection on which it is trained. A model with parameters learned from data could shed light on the flaws of the present key model as well as making the chroma models easily adapt to changes in the audio front-end. This may be especially useful when applying the basic model structure in different domains, e.g. chord extraction from MIDI, or figured bass extraction from Baroque recordings. We would like to extend our approach further and work towards a more complete model of music listening which includes beat detection, form, melody, and time signature.

\section{REFERENCES}

[1] R. Rawlins and N. E. Bahha, Jazzology. Hal Leonard Corporation, 2005.

[2] F. Mercury, J. Deacon, B. H. May, and R. M. Taylor, Greatest Hits II: Top Line and Chorus, B. M. E. Ltd., Ed. Queen Music Ltd./EMI Music Publishing, 1992.

[3] C. Raphael, "A graphical model for recognizing sung melodies," in Proceedings of the 2005 ISMIR Conference, London, UK, 2005 , pp. 658-663. 
[4] (2008) MIREX audio chord detection subtask, music information retrieval evaluation exchange. Available at http://www.music-ir. org/mirex/2008/index.php/Audio_Chord_Detection.

[5] R. Shepard, "Pitch perception and measurement," in Music, Cognition, and Computerized Sound: An Introduction to Psychoacoustics, P. Cook, Ed. MIT Press, 1999, pp. 149-165.

[6] T. Fujishima, "Real time chord recognition of musical sound: a system using Common Lisp Music," in Proceedings of the International Computer Music Conference (ICMC), 1999, pp. 464-467.

[7] E. Gomez, "Tonal Description of Audio Music Signals," Ph.D. dissertation, Universitat Pompeu Fabra, Barcelona, 2006.

[8] F. Kurth, M. Müller, C. Fremerey, Y. Chang, and M. Clausen, "Automated synchronization of scanned sheet music with audio recordings," in Proceedings of the 8th International Conference on Music Information Retrieval, ISMIR 2007, Vienna, Austria, 2007, pp. 261-266.

[9] H. Papadopoulos and G. Peeters, "Large-scale Study of Chord Estimation Algorithms Based on Chroma Representation and HMM," in International Workshop on Content-Based Multimedia Indexing, 2007, pp. 53-60.

[10] C. Harte and M. Sandler, "Automatic chord identifcation using a quantised chromagram," in Proceedings of 118th Convention. Audio Engineering Society, 2005.

[11] G. Peeters, "Chroma-based estimation of musical key from audiosignal analysis," in Proceedings of the 7th International Conference on Music Information Retrieval, ISMIR 2006, Victoria, Canada, 2006.

[12] M. P. Ryynänen and A. P. Klapuri, "Automatic Transcription of Melody, Bass Line, and Chords in Polyphonic Music," Computer Music Journal, vol. 32, no. 3, pp. 72-86, 2008.

[13] A. Camacho, "Detection of Pitched/Unpitched Sound Using Pitch Strength Clustering," in Proceedings of the 9th International Conference on Music Information Retrieval, ISMIR 2008, Philadelphia, USA, 2008, pp. 533-537.

[14] T. Yoshioka, T. Kitahara, K. Komatani, T. Ogata, and H. G. Okuno, "Automatic chord transcription with concurrent recognition of chord symbols and boundaries," in Proceedings of the 5th International Conference on Music Information Retrieval, ISMIR 2004, Barcelona, Spain, 2004, pp. 100-105.

[15] J. P. Bello and J. Pickens, "A Robust Mid-level Representation for Harmonic Content in Music Signals," in Proceedings of the 6th International Conference on Music Information Retrieval, ISMIR 2005, London, UK, 2005, pp. 304-311.

[16] J.-F. Paiement, D. Eck, and S. Bengio, "A probabilistic model for chord progressions," in Proceedings of the 6th International Conference on Music Information Retrieval, ISMIR 2005, London, UK, 2005, pp. 312-319.

[17] J. A. Burgoyne, L. Pugin, C. Kereliuk, and I. Fujinaga, "A CrossValidated Study of Modelling Strategies for Automatic Chord Recognition in Audio," in Proceedings of the 8th International Conference on Music Information Retrieval, ISMIR 2007, Vienna, Austria, 2007, pp. 251-254.

[18] K. Lee and M. Slaney, "Acoustic Chord Transcription and Key Extraction From Audio Using Key-Dependent HMMs Trained on Synthesized Audio," IEEE Transactions on Audio, Speech, and Language Processing, vol. 16, no. 2, pp. 291-301, February 2008.

[19] K. Noland and M. Sandler, "Key Estimation Using a Hidden Markov Model," in Proceedings of the 7th International Conference on Music Information Retrieval, ISMIR 2006, Victoria, Canada, 2006.

[20] M. Mauch and S. Dixon, "A Discrete Mixture Model for Chord Labelling," in Proceedings of the 9th International Conference on Music Information Retrieval, ISMIR 2008, Philadelphia, USA, 2008, pp. 45-50.

[21] H. Papadopoulos and G. Peeters, "Simultaneous estimation of chord progression and downbeats from an audio file," in Proceedings of the 2008 ICASSP Conference, 2008, pp. 121-124.

[22] R. J. Leistikow, "Bayesian Modeling of Musical Expectations via Maximum Entropy Stochastic Grammars," Ph.D. dissertation, Department of Music, Stanford University, June 2006.

[23] H. Thornburg, R. J. Leistikow, and J. Berger, "Melody Extraction and Musical Onset Detection via Probabilistic Models of Framewise STFT Peak Data," IEEE Transactions on Audio, Speech, and Language Processing, vol. 15, no. 4, pp. 1257-1272, 2007.

[24] K. Dressler and S. Streich, "Tuning Frequency Estimation Using Circular Statistics," in Proceedings of the 2007 ISMIR Conference, Vienna, Austria, 2007, pp. 357-360.

[25] F. Lerdahl and R. Jackendoff, A Generative Theory of Tonal Music. MIT Press, 1983.

[26] M. Davies, "Towards automatic rhythmic accompaniment," Ph.D. dissertation, Queen Mary University of London, London, UK, August 2007. [Online]. Available: http://www.elec.qmul.ac. uk/digitalmusic/papers/2007/Davies07-phdthesis.pdf

[27] K. P. Murphy, "Dynamic Bayesian networks: Representation, inference and learning," Ph.D. dissertation, University of California, Berkeley, 2002.

[28] X. Boyen and D. Koller, "Tractable inference for complex stochastic processes," in Proceedings of the Fourteenth Annual Conference on Uncertainty in Artificial Intelligence (UAI-98), 1998, pp. 33-42.

[29] K. P. Murphy, "The Bayes Net Toolbox for Matlab," Computing Science and Statistics, vol. 33, no. 2, pp. 1024-1034, 2001.

[30] C. Harte, M. Sandler, S. A. Abdallah, and E. Gomez, "Symbolic representation of musical chords: A proposed syntax for text annotations," in Proceedings of the 6th International Conference on Music Information Retrieval, ISMIR 2005, London, UK, 2005, pp. 66-71.

[31] C. L. Krumhansl, Cognitive Foundations of Musical Pitch. Oxford University Press, 1990.

[32] P. Westwood, Bass Bible: a world history of styles and techniques. AMA Verlag, 1997.

[33] Q. Huang and B. Dom, "Quantitative methods of evaluating image segmentation," in Proceedings of the International Conference on Image Processing, 1995, vol. 3, 1995, pp. 53-56.

[34] S. Abdallah, K. Noland, M. Sandler, M. Casey, and C. Rhodes, "Theory and evaluation of a Bayesian music structure extractor," in Proceedings of the 6th International Conference on Music Information Retrieval, ISMIR 2005, London, UK, 2005, pp. 420425.

[35] D. Hull, "Using statistical testing in the evaluation of retrieval experiments," in Proceedings of the 16th annual international ACM SIGIR conference on Research and development in information retrieval. ACM New York, USA, 1993, pp. 329-338.

[36] K. Noland and M. Sandler, "Influences of signal processing, tone profiles, and chord progressions on a model for estimating the musical key from audio," Computer Music Journal, vol. 33, no. 1, 2009. 